\title{
Properties on a subclass of univalent functions defined by using a multiplier transformation and Ruscheweyh derivative
}

\begin{abstract}
Alina Alb Lupaş
Abstract

In this paper we have introduced and studied the subclass $\mathcal{R J}(d, \alpha, \beta)$ of univalent functions defined by the linear operator $R I_{n, \lambda, l}^{\gamma} f(z)$ defined by using the Ruscheweyh derivative $R^{n} f(z)$ and multiplier transformation $I(n, \lambda, l) f(z)$, as $R I_{n, \lambda, l}^{\gamma}: \mathcal{A} \rightarrow \mathcal{A}, R I_{n, \lambda, l}^{\gamma} f(z)=(1-\gamma) R^{n} f(z)+$ $\gamma I(n, \lambda, l) f(z), z \in U$, where $\mathcal{A}_{n}=\left\{f \in \mathcal{H}(U): f(z)=z+a_{n+1} z^{n+1}+\right.$ $\ldots, z \in U\}$ is the class of normalized analytic functions with $\mathcal{A}_{1}=\mathcal{A}$. The main object is to investigate several properties such as coefficient estimates, distortion theorems, closure theorems, neighborhoods and the radii of starlikeness, convexity and close-to-convexity of functions belonging to the class $\mathcal{R J}(d, \alpha, \beta)$.
\end{abstract}

\section{Introduction}

Denote by $U$ the unit disc of the complex plane, $U=\{z \in \mathbb{C}:|z|<1\}$ and $\mathcal{H}(U)$ the space of holomorphic functions in $U$.

Let $\mathcal{A}_{n}=\left\{f \in \mathcal{H}(U): f(z)=z+a_{n+1} z^{n+1}+\ldots, z \in U\right\}$ with $\mathcal{A}_{1}=\mathcal{A}$.

Key Words: univalent function, Starlike functions, Convex functions, Distortion theorem.

2010 Mathematics Subject Classification: Primary 30C45, Secondary 30A20, 34A40.

Received: 3 May, 2014.

Revised: 24 June, 2014.

Accepted: 29 June, 2014. 
PROPERTIES OA SUBCLASS OF UNIVALENT FUNCTIONS DEFINED BY USING A MULTIPLIER TRANSFORMATION AND RUSCHEWEYH DERIVATIVE

Definition 1. (Ruscheweyh [20]) For $f \in \mathcal{A}, n \in \mathbb{N}$, the operator $R^{n}$ is defined by $R^{n}: \mathcal{A} \rightarrow \mathcal{A}$,

$$
\begin{aligned}
R^{0} f(z) & =f(z) \\
R^{1} f(z) & =z f^{\prime}(z), \ldots \\
(n+1) R^{n+1} f(z) & =z\left(R^{n} f(z)\right)^{\prime}+n R^{n} f(z), \quad z \in U .
\end{aligned}
$$

Remark 1. If $f \in \mathcal{A}, f(z)=z+\sum_{j=2}^{\infty} a_{j} z^{j}$, then

$$
R^{n} f(z)=z+\sum_{j=2}^{\infty} \frac{(n+j-1) !}{n !(j-1) !} a_{j} z^{j}, z \in U .
$$

Definition 2. For $f \in \mathcal{A}, n \in \mathbb{N}, \lambda, l \geq 0$, the operator $I(n, \lambda, l) f(z)$ is defined by the following infinite series

$$
I(n, \lambda, l) f(z)=z+\sum_{j=2}^{\infty}\left(\frac{\lambda(j-1)+l+1}{l+1}\right)^{n} a_{j} z^{j} .
$$

Remark 2. It follows from the above definition that

$$
\begin{aligned}
& \qquad(0, \lambda, l) f(z)=f(z), \\
& (l+1) I(n+1, \lambda, l) f(z)=(l+1-\lambda) I(n, \lambda, l) f(z)+\lambda z(I(n, \lambda, l) f(z))^{\prime}, \\
& z \in U .
\end{aligned}
$$

Remark 3. For $l=0, \lambda \geq 0$, the operator $D_{\lambda}^{n}=I(n, \lambda, 0)$ was introduced and studied by Al-Oboudi [16], which is reduced to the Sălăgean differential operator [21] for $\lambda=1$.

Definition 3. [7] Let $\gamma, \lambda, l \geq 0, n \in \mathbb{N}$. Denote by $R I_{n, \lambda, l}^{\gamma}$ the operator given by $R I_{n, \lambda, l}^{\gamma}: \mathcal{A} \rightarrow \mathcal{A}, \quad R I_{n, \lambda, l}^{\gamma} f(z)=(1-\gamma) R^{n} f(z)+\gamma I(n, \lambda, l) f(z), \quad z \in U$.

Remark 4. If $f \in \mathcal{A}, f(z)=z+\sum_{j=2}^{\infty} a_{j} z^{j}$, then $R I_{n, \lambda, l}^{\gamma} f(z)=z+\sum_{j=2}^{\infty}\left\{\gamma\left(\frac{1+\lambda(j-1)+l}{l+1}\right)^{n}+(1-\gamma) \frac{(n+j-1) !}{n !(j-1) !}\right\} a_{j} z^{j}, z \in U$.

This operator was studied also in [13], [14].

Remark 5. For $\alpha=0, R I_{m, \lambda, l}^{0} f(z)=R^{m} f(z)$, where $z \in U$ and for $\alpha=1$, $R I_{m, \lambda, l}^{1} f(z)=I(m, \lambda, l) f(z)$, where $z \in U$, which was studied in [3], [4], [10], [9]. For $l=0$, we obtain $R I_{m, \lambda, 0}^{\alpha} f(z)=R D_{\lambda, \alpha}^{m} f(z)$ which was studied in [5], [6], [11], [12], [17], [18] and for $l=0$ and $\lambda=1$, we obtain $R I_{m, 1,0}^{\alpha} f(z)=$ $L_{\alpha}^{m} f(z)$ which was studied in [1], [2], [8], [15]. 
PROPERTIES OA SUBCLASS OF UNIVALENT FUNCTIONS DEFINED BY USING A MULTIPLIER TRANSFORMATION AND RUSCHEWEYH DERIVATIVE

We follow the works of A.R. Juma and H. Ziraz [19].

Definition 4. Let the function $f \in \mathcal{A}$. Then $f(z)$ is said to be in the class $\mathcal{R J}(d, \alpha, \beta)$ if it satisfies the following criterion:

$$
\left|\frac{1}{d}\left(\frac{\left.z\left(R I_{n, \lambda, l}^{\gamma} f(z)\right)^{\prime}+\alpha z^{2} R I_{n, \lambda, l}^{\gamma} f(z)\right)^{\prime \prime}}{(1-\alpha) R I_{n, \lambda, l}^{\gamma} f(z)+\alpha z\left(R I_{n, \lambda, l}^{\gamma} f(z)\right)^{\prime}}-1\right)\right|<\beta,
$$

where $d \in \mathbb{C}-\{0\}, 0 \leq \alpha \leq 1,0<\beta \leq 1, z \in U$

In this paper we shall first deduce a necessary and sufficient condition for a function $f(z)$ to be in the class $\mathcal{R J}(d, \alpha, \beta)$. Then obtain the distortion and growth theorems, closure theorems, neighborhood and radii of univalent starlikeness, convexity and close-to-convexity of order $\delta, 0 \leq \delta<1$, for these functions.

\section{Coefficient Inequality}

Theorem 1. Let the function $f \in \mathcal{A}$. Then $f(z)$ is said to be in the class $\mathcal{R J}(d, \alpha, \beta)$ if and only if

$$
\begin{gathered}
\sum_{j=2}^{\infty}(1+\alpha(j-1))(j-1+\beta|d|) . \\
\left\{\gamma\left(\frac{1+\lambda(j-1)+l}{l+1}\right)^{n}+(1-\gamma) \frac{(n+j-1) !}{n !(j-1) !}\right\} a_{j} \leq \beta|d|,
\end{gathered}
$$

where $d \in \mathbb{C}-\{0\}, 0 \leq \alpha \leq 1,0<\beta \leq 1, z \in U$.

Proof. Let $f(z) \in \mathcal{R J}(d, \alpha, \beta)$. Assume that inequality (2) holds true. Then we find that

$$
\begin{gathered}
\left|\frac{z\left(R I_{n, \lambda, l}^{\gamma} f(z)\right)^{\prime}+\alpha z^{2}\left(R I_{n, \lambda, l}^{\gamma} f(z)\right)^{\prime \prime}}{(1-\alpha) R I_{n, \lambda, l}^{\gamma} f(z)+\alpha z\left(R I_{n, \lambda, l}^{\gamma} f(z)\right)^{\prime}}-1\right| \\
=\left|\frac{\sum_{j=2}^{\infty}(1+\alpha(j-1))(j-1)\left\{\gamma\left(\frac{1+\lambda(j-1)+l}{l+1}\right)^{n}+(1-\gamma) \frac{(n+j-1) !}{n !(j-1) !}\right\} a_{j} z^{j}}{z+\sum_{j=2}^{\infty}(1+\alpha(j-1))\left\{\gamma\left(\frac{1+\lambda(j-1)+l}{l+1}\right)^{n}+(1-\gamma) \frac{(n+j-1) !}{n !(j-1) !}\right\} a_{j} z^{j}}\right| \\
\leq \frac{\sum_{j=2}^{\infty}(1+\alpha(j-1))(j-1)\left\{\gamma\left(\frac{1+\lambda(j-1)+l}{l+1}\right)^{n}+(1-\gamma) \frac{(n+j-1) !}{n !(j-1) !}\right\} a_{j}|z|^{j-1}}{1-\sum_{j=2}^{\infty}(1+\alpha(j-1))\left\{\gamma\left(\frac{1+\lambda(j-1)+l}{l+1}\right)^{n}+(1-\gamma) \frac{(n+j-1) !}{n !(j-1) !}\right\} a_{j}|z|^{j-1}} \\
<\beta|d| .
\end{gathered}
$$


PROPERTIES OA SUBCLASS OF UNIVALENT FUNCTIONS DEFINED BY USING A MULTIPLIER TRANSFORMATION AND RUSCHEWEYH

Choosing values of $z$ on real axis and letting $z \rightarrow 1^{-}$, we have

$$
\begin{gathered}
\sum_{j=2}^{\infty}(1+\alpha(j-1))(j-1+\beta|d|)\left\{\gamma\left(\frac{1+\lambda(j-1)+l}{l+1}\right)^{n}+(1-\gamma) \frac{(n+j-1) !}{n !(j-1) !}\right\} a_{j} \\
\leq \beta|d| .
\end{gathered}
$$

Conversely, assume that $f(z) \in \mathcal{R J}(d, \alpha, \beta)$, then we get the following inequality

$$
\begin{gathered}
\operatorname{Re}\left\{\frac{z\left(R I_{n, \lambda, l}^{\gamma} f(z)\right)^{\prime}+\alpha z^{2}\left(R I_{n, \lambda, l}^{\gamma} f(z)\right)^{\prime \prime}}{(1-\alpha) R I_{n, \lambda, l}^{\gamma} f(z)+\alpha z\left(R I_{n, \lambda, l}^{\gamma} f(z)\right)^{\prime}}-1 \mid\right\}>-\beta|d| \\
\operatorname{Re}\left\{\frac{z+\sum_{j=2}^{\infty} j(1+\alpha(j-1))\left\{\gamma\left(\frac{1+\lambda(j-1)+l}{l+1}\right)^{n}+(1-\gamma) \frac{(n+j-1) !}{n !(j-1) !}\right\} a_{j} z^{j}}{z+\sum_{j=2}^{\infty}(1+\alpha(j-1))\left\{\gamma\left(\frac{1+\lambda(j-1)+l}{l+1}\right)^{n}+(1-\gamma) \frac{(n+j-1) !}{n !(j-1) !}\right\} a_{j} z^{j}}-1+\beta|d|\right\}>0 \\
\operatorname{Re} \frac{\beta|d| z+\sum_{j=2}^{\infty}(1+\alpha(j-1))(j-1+\beta|d|)\left\{\gamma\left(\frac{1+\lambda(j-1)+l}{l+1}\right)^{n}+(1-\gamma) \frac{(n+j-1) !}{n !(j-1) !}\right\} a_{j} z^{j}}{z+\sum_{j=2}^{\infty}(1+\alpha(j-1))\left\{\gamma\left(\frac{1+\lambda(j-1)+l}{l+1}\right)^{n}+(1-\gamma) \frac{(n+j-1) !}{n !(j-1) !}\right\} a_{j} z^{j}}>0 .
\end{gathered}
$$

Since $\operatorname{Re}\left(-e^{i \theta}\right) \geq-\left|e^{i \theta}\right|=-1$, the above inequality reduces to

$$
\frac{\beta|d| r-\sum_{j=2}^{\infty}(1+\alpha(j-1))(j-1+\beta|d|)\left\{\gamma\left(\frac{1+\lambda(j-1)+l}{l+1}\right)^{n}+(1-\gamma) \frac{(n+j-1) !}{n !(j-1) !}\right\} a_{j} r^{j}}{r-\sum_{j=2}^{\infty}(1+\alpha(j-1))\left\{\gamma\left(\frac{1+\lambda(j-1)+l}{l+1}\right)^{n}+(1-\gamma) \frac{(n+j-1) !}{n !(j-1) !}\right\} a_{j} r^{j}}>0 .
$$

Letting $r \rightarrow 1^{-}$and by the mean value theorem we have desired inequality (2).

This completes the proof of Theorem 1

Corollary 1. Let the function $f \in \mathcal{A}$ be in the class $\mathcal{R J}(d, \alpha, \beta)$. Then

$$
a_{j} \leq \frac{\beta|d|}{(1+\alpha(j-1))(j-1+\beta|d|)\left\{\gamma\left(\frac{1+\lambda(j-1)+l}{l+1}\right)^{n}+(1-\gamma) \frac{(n+j-1) !}{n !(j-1) !}\right\}}, \quad j \geq 2
$$

\section{Distortion Theorems}

Theorem 2. Let the function $f \in \mathcal{A}$ be in the class $\mathcal{R J}(d, \alpha, \beta)$. Then for $|z|=r<1$, we have

$$
r-\frac{\beta|d|}{(1+\alpha)(1+\beta|d|)\left[\gamma\left(\frac{1+\lambda+l}{l+1}\right)^{n}+(1-\gamma)(n+1)\right]} r^{2} \leq|f(z)|
$$


PROPERTIES OA SUBCLASS OF UNIVALENT FUNCTIONS DEFINED BY USING A MULTIPLIER TRANSFORMATION AND RUSCHEWEYH

$$
\leq r+\frac{\beta|d|}{(1+\alpha)(1+\beta|d|)\left[\gamma\left(\frac{1+\lambda+l}{l+1}\right)^{n}+(1-\gamma)(n+1)\right]} r^{2} .
$$

The result is sharp for the function $f(z)$ given by

$$
f(z)=z+\frac{\beta|d|}{(1+\alpha)(1+\beta|d|)\left[\gamma\left(\frac{1+\lambda+l}{l+1}\right)^{n}+(1-\gamma)(n+1)\right]} z^{2} .
$$

Proof. Given that $f(z) \in \mathcal{R J}(d, \alpha, \beta)$, from the equation (2) and since

$$
(1+\alpha)(1+\beta|d|)\left[\gamma\left(\frac{1+\lambda+l}{l+1}\right)^{n}+(1-\gamma)(n+1)\right]
$$

is non decreasing and positive for $j \geq 2$, then we have

$$
\begin{gathered}
(1+\alpha)(1+\beta|d|)\left[\gamma\left(\frac{1+\lambda+l}{l+1}\right)^{n}+(1-\gamma)(n+1)\right] \sum_{j=2}^{\infty} a_{j} \leq \\
\sum_{j=2}^{\infty}(1+\alpha(j-1))(j-1+\beta|d|)\left\{\gamma\left(\frac{1+\lambda(j-1)+l}{l+1}\right)^{n}+(1-\gamma) \frac{(n+j-1) !}{n !(j-1) !}\right\} a_{j} \\
\leq \beta|d|,
\end{gathered}
$$

which is equivalent to,

$$
\sum_{j=2}^{\infty} a_{j} \leq \frac{\beta|d|}{(1+\alpha)(1+\beta|d|)\left[\gamma\left(\frac{1+\lambda+l}{l+1}\right)^{n}+(1-\gamma)(n+1)\right]} .
$$

Using (3), we obtain

$$
\begin{gathered}
f(z)=z+\sum_{j=2}^{\infty} a_{j} z^{j} \\
|f(z)| \leq|z|+\sum_{j=2}^{\infty} a_{j}|z|^{j} \leq r+\sum_{j=2}^{\infty} a_{j} r^{j} \leq r+r^{2} \sum_{j=2}^{\infty} a_{j} \\
\leq r+\frac{\beta|d|}{(1+\alpha)(1+\beta|d|)\left[\gamma\left(\frac{1+\lambda+l}{l+1}\right)^{n}+(1-\gamma)(n+1)\right]} r^{2} .
\end{gathered}
$$

Similarly,

$$
|f(z)| \geq r^{2}-\frac{\beta|d|}{(1+\alpha)(1+\beta|d|)\left[\gamma\left(\frac{1+\lambda+l}{l+1}\right)^{n}+(1-\gamma)(n+1)\right]} r^{2} .
$$

This completes the proof of Theorem 2 . 
PROPERTIES OA SUBCLASS OF UNIVALENT FUNCTIONS DEFINED BY USING A MULTIPLIER TRANSFORMATION AND RUSCHEWEYH

Theorem 3. Let the function $f \in \mathcal{A}$ be in the class $\mathcal{R J}(d, \alpha, \beta)$. Then for $|z|=r<1$, we have

$$
\begin{gathered}
-\frac{2 \beta|d|}{(1+\alpha)(1+\beta|d|)\left[\gamma\left(\frac{1+\lambda+l}{l+1}\right)^{n}+(1-\gamma)(n+1)\right]} r \leq\left|f^{\prime}(z)\right| \\
\quad \leq \frac{2 \beta|d|}{(1+\alpha)(1+\beta|d|)\left[\gamma\left(\frac{1+\lambda+l}{l+1}\right)^{n}+(1-\gamma)(n+1)\right]} r .
\end{gathered}
$$

The result is sharp for the function $f(z)$ given by

$$
f(z)=z+\frac{\beta|d|}{(1+\alpha)(1+\beta|d|)\left[\gamma\left(\frac{1+\lambda+l}{l+1}\right)^{n}+(1-\gamma)(n+1)\right]} z^{2} .
$$

Proof. From (3)

$$
\begin{gathered}
f^{\prime}(z)=1+\sum_{j=2}^{\infty} j a_{j} z^{j-1} \\
\left|f^{\prime}(z)\right| \leq 1-\sum_{j=2}^{\infty} j a_{j}|z|^{j-1} \leq 1+\sum_{j=2}^{\infty} j a_{j} r^{j-1} \\
\leq 1+\frac{2 \beta|d|}{(1+\alpha)(1+\beta|d|)\left[\gamma\left(\frac{1+\lambda+l}{l+1}\right)^{n}+(1-\gamma)(n+1)\right]} r .
\end{gathered}
$$

Similarly,

$$
\left|f^{\prime}(z)\right| \geq 1-\frac{2 \beta|d|}{(1+\alpha)(1+\beta|d|)\left[\gamma\left(\frac{1+\lambda+l}{l+1}\right)^{n}+(1-\gamma)(n+1)\right]} r .
$$

This completes the proof of Theorem 3 .

\section{Closure Theorems}

Theorem 4. Let the functions $f_{k}, k=1,2, \ldots, m$, defined by

$$
f_{k}(z)=z+\sum_{j=2}^{\infty} a_{j, k} z^{j}, \quad a_{j, k} \geq 0,
$$

be in the class $\mathcal{R J}(d, \alpha, \beta)$. Then the function $h(z)$ defined by

$$
h(z)=\sum_{k=1}^{m} \mu_{k} f_{k}(z), \quad \mu_{k} \geq 0,
$$


PROPERTIES OA SUBCLASS OF UNIVALENT FUNCTIONS DEFINED BY USING A MULTIPLIER TRANSFORMATION AND RUSCHEWEYH

is also in the class $\mathcal{R J}(d, \alpha, \beta)$, where

$$
\sum_{k=1}^{m} \mu_{k}=1
$$

Proof. We can write

$$
h(z)=\sum_{k=1}^{m} \mu_{m} z+\sum_{k=1}^{m} \sum_{j=2}^{\infty} \mu_{k} a_{j, k} z^{j}=z+\sum_{j=2}^{\infty} \sum_{k=1}^{m} \mu_{k} a_{j, k} z^{j} .
$$

Furthermore, since the functions $f_{k}(z), k=1,2, \ldots, m$, are in the class $\mathcal{R J}(d, \alpha, \beta)$, then from Theorem 1 we have

$$
\begin{gathered}
\sum_{j=2}^{\infty}(1+\alpha(j-1))(j-1+\beta|d|) . \\
\left\{\gamma\left(\frac{1+\lambda(j-1)+l}{l+1}\right)^{n}+(1-\gamma) \frac{(n+j-1) !}{n !(j-1) !}\right\} a_{j, k} \leq \beta|d| .
\end{gathered}
$$

Thus it is enough to prove that

$$
\begin{gathered}
\sum_{j=2}^{\infty}(1+\alpha(j-1))(j-1+\beta|d|) . \\
\left\{\gamma\left(\frac{1+\lambda(j-1)+l}{l+1}\right)^{n}+(1-\gamma) \frac{(n+j-1) !}{n !(j-1) !}\right\}\left(\sum_{k=1}^{m} \mu_{k} a_{j, k}\right)= \\
\left\{\gamma\left(\frac{1+\lambda(j-1)+l}{l+1}\right)^{n}+(1-\gamma) \frac{(n+j-1) !}{n !(j-1) !}\right\} a_{j, k} \\
\leq \sum_{k=1}^{m} \mu_{k} \beta|d|=\beta|d| .
\end{gathered}
$$

Hence the proof is complete.

Corollary 2. Let the functions $f_{k}, k=1,2$, defined by (4) be in the class $\mathcal{R J}(d, \alpha, \beta)$. Then the function $h(z)$ defined by

$$
h(z)=(1-\zeta) f_{1}(z)+\zeta f_{2}(z), \quad 0 \leq \zeta \leq 1,
$$

is also in the class $\mathcal{R J}(d, \alpha, \beta)$. 
PROPERTIES OA SUBCLASS OF UNIVALENT FUNCTIONS DEFINED BY USING A MULTIPLIER TRANSFORMATION AND RUSCHEWEYH

Theorem 5. Let

$$
f_{1}(z)=z,
$$

and

$f_{j}(z)=z+\frac{\beta|d|}{(1+\alpha(j-1))(j-1+\beta|d|)\left\{\gamma\left(\frac{1+\lambda(j-1)+l}{l+1}\right)^{n}+(1-\gamma) \frac{(n+j-1) !}{n !(j-1) !}\right\}} z^{j}$,

$j \geq 2$.

Then the function $f(z)$ is in the class $\mathcal{R J}(d, \alpha, \beta)$ if and only if it can be expressed in the form:

$$
f(z)=\mu_{1} f_{1}(z)+\sum_{j=2}^{\infty} \mu_{j} f_{j}(z)
$$

where $\mu_{1} \geq 0, \mu_{j} \geq 0, j \geq 2$ and $\mu_{1}+\sum_{j=2}^{\infty} \mu_{j}=1$.

Proof. Assume that $f(z)$ can be expressed in the form

$$
\begin{gathered}
f(z)=\mu_{1} f_{1}(z)+\sum_{j=2}^{\infty} \mu_{j} f_{j}(z)= \\
z+\sum_{j=2}^{\infty} \frac{\beta|d|}{(1+\alpha(j-1))(j-1+\beta|d|)\left\{\gamma\left(\frac{1+\lambda(j-1)+l}{l+1}\right)^{n}+(1-\gamma) \frac{(n+j-1) !}{n !(j-1) !}\right\}} \mu_{j} z^{j} .
\end{gathered}
$$

Thus

$$
\begin{gathered}
\sum_{j=2}^{\infty} \frac{(1+\alpha(j-1))(j-1+\beta|d|)\left\{\gamma\left(\frac{1+\lambda(j-1)+l}{l+1}\right)^{n}+(1-\gamma) \frac{(n+j-1) !}{n !(j-1) !}\right\}}{\beta|d|} . \\
\frac{\beta|d|}{(1+\alpha(j-1))(j-1+\beta|d|)\left\{\gamma\left(\frac{1+\lambda(j-1)+l}{l+1}\right)^{n}+(1-\gamma) \frac{(n+j-1) !}{n !(j-1) !}\right\}} \mu_{j} \\
=\sum_{j=2}^{\infty} \mu_{j}=1-\mu_{1} \leq 1 .
\end{gathered}
$$

Hence $f(z) \in \mathcal{R J}(d, \alpha, \beta)$.

Conversely, assume that $f(z) \in \mathcal{R J}(d, \alpha, \beta)$.

Setting

$$
\mu_{j}=\frac{(1+\alpha(j-1))(j-1+\beta|d|)\left\{\gamma\left(\frac{1+\lambda(j-1)+l}{l+1}\right)^{n}+(1-\gamma) \frac{(n+j-1) !}{n !(j-1) !}\right\}}{\beta|d|} a_{j},
$$


PROPERTIES OA SUBCLASS OF UNIVALENT FUNCTIONS DEFINED

BY USING A MULTIPLIER TRANSFORMATION AND RUSCHEWEYH

since

$$
\mu_{1}=1-\sum_{j=2}^{\infty} \mu_{j}
$$

Thus

$$
f(z)=\mu_{1} f_{1}(z)+\sum_{j=2}^{\infty} \mu_{j} f_{j}(z) .
$$

Hence the proof is complete.

Corollary 3. The extreme points of the class $\mathcal{R J}(d, \alpha, \beta)$ are the functions

$$
f_{1}(z)=z,
$$

and

$f_{j}(z)=z+\frac{\beta|d|}{(1+\alpha(j-1))(j-1+\beta|d|)\left\{\gamma\left(\frac{1+\lambda(j-1)+l}{l+1}\right)^{n}+(1-\gamma) \frac{(n+j-1) !}{n !(j-1) !}\right\}} z^{j}$,

$j \geq 2$.

\section{Inclusion and Neighborhood Results}

We define the $\delta$ - neighborhood of a function $f(z) \in \mathcal{A}$ by

$$
N_{\delta}(f)=\left\{g \in \mathcal{A}: g(z)=z+\sum_{j=2}^{\infty} b_{j} z^{j} \text { and } \sum_{j=2}^{\infty} j\left|a_{j}-b_{j}\right| \leq \delta\right\} .
$$

In particular, for $e(z)=z$

$$
N_{\delta}(e)=\left\{g \in \mathcal{A}: g(z)=z+\sum_{j=2}^{\infty} b_{j} z^{j} \text { and } \sum_{j=2}^{\infty} j\left|b_{j}\right| \leq \delta\right\} .
$$

Furthermore, a function $f \in \mathcal{A}$ is said to be in the class $\mathcal{R J}^{\xi}(d, \alpha, \beta)$ if there exists a function $h(z) \in \mathcal{R J}(d, \alpha, \beta)$ such that

$$
\left|\frac{f(z)}{h(z)}-1\right|<1-\xi, \quad z \in U, \quad 0 \leq \xi<1 .
$$

Theorem 6. If

$$
\left\{\gamma\left(\frac{1+\lambda(j-1)+l}{l+1}\right)^{n}+(1-\gamma) \frac{(n+j-1) !}{n !(j-1) !}\right\}
$$


PROPERTIES OA SUBCLASS OF UNIVALENT FUNCTIONS DEFINED BY USING A MULTIPLIER TRANSFORMATION AND RUSCHEWEYH

$$
\geq\left[\gamma\left(\frac{1+\lambda+l}{l+1}\right)^{n}+(1-\gamma)(n+1)\right], \quad j \geq 2
$$

and

$$
\delta=\frac{2 \beta|d|}{(1+\alpha)(1+\beta|d|)\left[\gamma\left(\frac{1+\lambda+l}{l+1}\right)^{n}+(1-\gamma)(n+1)\right]}
$$

then

$$
\mathcal{R J}(d, \alpha, \beta) \subset N_{\delta}(e) .
$$

Proof. Let $f \in \mathcal{R J}(d, \alpha, \beta)$. Then in view of assertion (2) of Theorem 1 and the condition

$\left\{\gamma\left(\frac{1+\lambda(j-1)+l}{l+1}\right)^{n}+(1-\gamma) \frac{(n+j-1) !}{n !(j-1) !}\right\} \geq\left[\gamma\left(\frac{1+\lambda+l}{l+1}\right)^{n}+(1-\gamma)(n+1)\right]$ for $j \geq 2$, we get

$$
\begin{gathered}
(1+\alpha)(1+\beta|d|)\left[\gamma\left(\frac{1+\lambda+l}{l+1}\right)^{n}+(1-\gamma)(n+1)\right] \sum_{j=2}^{\infty} a_{j} \leq \\
\sum_{j=2}^{\infty}(1+\alpha(j-1))(j-1+\beta|d|)\left\{\gamma\left(\frac{1+\lambda(j-1)+l}{l+1}\right)^{n}+(1-\gamma) \frac{(n+j-1) !}{n !(j-1) !}\right\} a_{j} \\
\leq \beta|d|,
\end{gathered}
$$

which implise

$$
\sum_{j=2}^{\infty} a_{j} \leq \frac{\beta|d|}{(1+\alpha)(1+\beta|d|)\left[\gamma\left(\frac{1+\lambda+l}{l+1}\right)^{n}+(1-\gamma)(n+1)\right]}
$$

Applying assertion (2) of Theorem 1 in conjunction with (8), we obtain

$$
\begin{gathered}
(1+\alpha)(1+\beta|d|)\left[\gamma\left(\frac{1+\lambda+l}{l+1}\right)^{n}+(1-\gamma)(n+1)\right] \sum_{j=2}^{\infty} a_{j} \leq \beta|d|, \\
2(1+\alpha)(1+\beta|d|)\left[\gamma\left(\frac{1+\lambda+l}{l+1}\right)^{n}+(1-\gamma)(n+1)\right] \sum_{j=2}^{\infty} a_{j} \leq 2 \beta|d| \\
\sum_{j=2}^{\infty} j a_{j} \leq \frac{2 \beta|d|}{(1+\alpha)(1+\beta|d|)\left[\gamma\left(\frac{1+\lambda+l}{l+1}\right)^{n}+(1-\gamma)(n+1)\right]}=\delta,
\end{gathered}
$$

by virtue of (5), we have $f \in N_{\delta}(e)$.

This completes the proof of the Theorem 6 . 
PROPERTIES OA SUBCLASS OF UNIVALENT FUNCTIONS DEFINED BY USING A MULTIPLIER TRANSFORMATION AND RUSCHEWEYH

Theorem 7. If $h \in \mathcal{R J}(d, \alpha, \beta)$ and

$$
\xi=1-\frac{\delta}{2} \frac{(1+\alpha)(1+\beta|d|)\left[\gamma\left(\frac{1+\lambda+l}{l+1}\right)^{n}+(1-\gamma)(n+1)\right]}{(1+\alpha)(1+\beta|d|)\left[\gamma\left(\frac{1+\lambda+l}{l+1}\right)^{n}+(1-\gamma)(n+1)\right]-\beta|d|},
$$

then

$$
N_{\delta}(h) \subset \mathcal{R J}^{\xi}(d, \alpha, \beta) .
$$

Proof. Suppose that $f \in N_{\delta}(h)$, we then find from (5) that

$$
\sum_{j=2}^{\infty} j\left|a_{j}-b_{j}\right| \leq \delta
$$

which readily implies the following coefficient inequality

$$
\sum_{j=2}^{\infty}\left|a_{j}-b_{j}\right| \leq \frac{\delta}{2} .
$$

Next, since $h \in \mathcal{R J}(d, \alpha, \beta)$ in the view of (8), we have

$$
\sum_{j=2}^{\infty} b_{j} \leq \frac{\beta|d|}{(1+\alpha)(1+\beta|d|)\left[\gamma\left(\frac{1+\lambda+l}{l+1}\right)^{n}+(1-\gamma)(n+1)\right]} .
$$

Using 10) and (11), we get

$$
\begin{gathered}
\left|\frac{f(z)}{h(z)}-1\right| \leq \frac{\sum_{j=2}^{\infty}\left|a_{j}-b_{j}\right|}{1-\sum_{j=2}^{\infty} b_{j}} \leq \frac{\delta}{2\left(1-\frac{\beta|d|}{(1+\alpha)(1+\beta|d|)\left[\gamma\left(\frac{1+\lambda+l}{l+1}\right)^{n}+(1-\gamma)(n+1)\right]}\right)} \\
\quad \leq \frac{\delta}{2} \frac{(1+\alpha)(1+\beta|d|)\left[\gamma\left(\frac{1+\lambda+l}{l+1}\right)^{n}+(1-\gamma)(n+1)\right]}{(1+\alpha)(1+\beta|d|)\left[\gamma\left(\frac{1+\lambda+l}{l+1}\right)^{n}+(1-\gamma)(n+1)\right]-\beta|d|}=1-\xi,
\end{gathered}
$$

provided that $\xi$ is given by (9), thus by condition (7), $f \in \mathcal{R J}^{\xi}(d, \alpha, \beta)$, where $\xi$ is given by $(9)$.

\section{Radii of Starlikeness, Convexity and Close-to-Convexity}

Theorem 8. Let the function $f \in \mathcal{A}$ be in the class $\mathcal{R J}(d, \alpha, \beta)$. Then $f$ is univalent starlike of order $\delta, 0 \leq \delta<1$, in $|z|<r_{1}$, where

$$
r_{1}=
$$


PROPERTIES OA SUBCLASS OF UNIVALENT FUNCTIONS DEFINED BY USING A MULTIPLIER TRANSFORMATION AND RUSCHEWEYH DERIVATIVE

$\inf _{j}\left\{\frac{(1-\delta)(1+\alpha(j-1))(j-1+\beta|d|)\left\{\gamma\left(\frac{1+\lambda(j-1)+l}{l+1}\right)^{n}+(1-\gamma) \frac{(n+j-1) !}{n !(j-1) !}\right\}}{\beta|d|(1-\delta)}\right\}^{\frac{1}{j-1}}$.

The result is sharp for the function $f(z)$ given by

$$
f_{j}(z)=z+\frac{\beta|d|}{(1+\alpha(j-1))(j-1+\beta|d|)\left\{\gamma\left(\frac{1+\lambda(j-1)+l}{l+1}\right)^{n}+(1-\gamma) \frac{(n+j-1) !}{n !(j-1) !}\right\}} z^{j},
$$

$j \geq 2$.

Proof. It suffices to show that

$$
\left|\frac{z f^{\prime}(z)}{f(z)}-1\right| \leq 1-\delta, \quad|z|<r_{1} .
$$

Since

$$
\left|\frac{z f^{\prime}(z)}{f(z)}-1\right|=\left|\frac{\sum_{j=2}^{\infty}(j-1) a_{j} z^{j-1}}{1+\sum_{j=2}^{\infty} a_{j} z^{k-1}}\right| \mid \leq \frac{\sum_{j=2}^{\infty}(j-1) a_{j}|z|^{j-1}}{1-\sum_{j=2}^{\infty} a_{j}|z|^{j-1}} .
$$

To prove the theorem, we must show that

$$
\frac{\sum_{j=2}^{\infty}(j-1) a_{j}|z|^{j-1}}{1-\sum_{j=2}^{\infty} a_{j}|z|^{j-1}} \leq 1-\delta
$$

It is equivalent to

$$
\sum_{j=2}^{\infty}(j-\delta) a_{j}|z|^{j-1} \leq 1-\delta
$$

using Theorem 1, we obtain

$$
|z| \leq\left\{\frac{(1-\delta)(1+\alpha(j-1))(j-1+\beta|d|)\left\{\gamma\left(\frac{1+\lambda(j-1)+l}{l+1}\right)^{n}+(1-\gamma) \frac{(n+j-1) !}{n !(j-1) !}\right\}}{\beta|d|(1-\delta)}\right\}^{\frac{1}{j-1}} .
$$

Hence the proof is complete.

Theorem 9. Let the function $f \in \mathcal{A}$ be in the class $\mathcal{R J}(d, \alpha, \beta)$. Then $f$ is univalent convex of order $\delta, 0 \leq \delta \leq 1$, in $|z|<r_{2}$, where

$$
r_{2}=\inf _{j}\left\{\frac{(1-\delta)(1+\alpha(j-1))(j-1+\beta|d|)\left\{\gamma\left(\frac{1+\lambda(j-1)+l}{l+1}\right)^{n}+(1-\gamma) \frac{(n+j-1) !}{n !(j-1) !}\right\}}{2(j-\delta) \beta|d|}\right\}^{\frac{1}{k-p}} .
$$

The result is sharp for the function $f(z)$ given by

$$
\begin{gathered}
f_{j}(z)=z+\frac{\beta|d|}{(1+\alpha(j-1))(j-1+\beta|d|)\left\{\gamma\left(\frac{1+\lambda(j-1)+l}{l+1}\right)^{n}+(1-\gamma) \frac{(n+j-1) !}{n !(j-1) !}\right\}} z^{j}, \\
j \geq 2 .
\end{gathered}
$$


PROPERTIES OA SUBCLASS OF UNIVALENT FUNCTIONS DEFINED BY USING A MULTIPLIER TRANSFORMATION AND RUSCHEWEYH

Proof. It suffices to show that

$$
\left|\frac{z f^{\prime \prime}(z)}{\left.f^{\prime}(z)\right)}\right| \leq 1-\delta, \quad|z|<r_{2}
$$

Since

$$
\left|\frac{z f^{\prime \prime}(z)}{f^{\prime}(z)}\right|=\left|\frac{\sum_{j=2}^{\infty} j(j-1) a_{j} z^{j-1}}{1+\sum_{j=2}^{\infty} j a_{j} z^{j-1}}\right| \leq \frac{\sum_{j=2}^{\infty} j(j-1) a_{j}|z|^{j-1}}{1-\sum_{j=2}^{\infty} j a_{j}|z|^{j-1}} .
$$

To prove the theorem, we must show that

$$
\begin{gathered}
\frac{\sum_{j=2}^{\infty} j(j-1) a_{j}|z|^{j-1}}{1-\sum_{j=2}^{\infty} j a_{j}|z|^{j-1}} \leq 1-\delta, \\
\sum_{j=2}^{\infty} j(j-\delta) a_{j}|z|^{j-1} \leq 1-\delta,
\end{gathered}
$$

using Theorem 1, we obtain

$$
|z|^{j-1} \leq \frac{(1-\delta)(1+\alpha(j-1))(j-1+\beta|d|)\left\{\gamma\left(\frac{1+\lambda(j-1)+l}{l+1}\right)^{n}+(1-\gamma) \frac{(n+j-1) !}{n !(j-1) !}\right\}}{2(j-\delta) \beta|d|},
$$

or

$$
|z| \leq\left\{\frac{(1-\delta)(1+\alpha(j-1))(j-1+\beta|d|)\left\{\gamma\left(\frac{1+\lambda(j-1)+l}{l+1}\right)^{n}+(1-\gamma) \frac{(n+j-1) !}{n !(j-1) !}\right\}}{2(j-\delta) \beta|d|}\right\}^{\frac{1}{j-1}} .
$$

Hence the proof is complete.

Theorem 10. Let the function $f \in \mathcal{A}$ be in the class $\mathcal{R J}(d, \alpha, \beta)$. Then $f$ is univalent close-to-convex of order $\delta, 0 \leq \delta<1$, in $|z|<r_{3}$, where

$$
r_{3}=\inf _{j}\left\{\frac{(1-\delta)(1+\alpha(j-1))(j-1+\beta|d|)\left\{\gamma\left(\frac{1+\lambda(j-1)+l}{l+1}\right)^{n}+(1-\gamma) \frac{(n+j-1) !}{n !(j-1) !}\right\}}{j \beta|d|}\right\}^{\frac{1}{j-1}} .
$$

The result is sharp for the function $f(z)$ given by (12).

Proof. It suffices to show that

$$
\left|f^{\prime}(z)-1\right| \leq 1-\delta, \quad|z|<r_{3} .
$$

Then

$$
\left|f^{\prime}(z)-1\right|=\left|\sum_{j=2}^{\infty} j a_{j} z^{j-1}\right| \leq \sum_{j=2}^{\infty} j a_{j}|z|^{j-1}
$$


PROPERTIES OA SUBCLASS OF UNIVALENT FUNCTIONS DEFINED BY USING A MULTIPLIER TRANSFORMATION AND RUSCHEWEYH DERIVATIVE

Thus $\left|f^{\prime}(z)-1\right| \leq 1-\delta$ if $\sum_{j=2}^{\infty} \frac{j a_{j}}{1-\delta}|z|^{j-1} \leq 1$. Using Theorem 1 , the above inequality holds true if

$$
|z|^{j-1} \leq \frac{(1-\delta)(1+\alpha(j-1))(j-1+\beta|d|)\left\{\gamma\left(\frac{1+\lambda(j-1)+l}{l+1}\right)^{n}+(1-\gamma) \frac{(n+j-1) !}{n !(j-1) !}\right\}}{j \beta|d|}
$$

or

$$
|z| \leq\left\{\frac{(1-\delta)(1+\alpha(j-1))(j-1+\beta|d|)\left\{\gamma\left(\frac{1+\lambda(j-1)+l}{l+1}\right)^{n}+(1-\gamma) \frac{(n+j-1) !}{n !(j-1) !}\right\}}{j \beta|d|}\right\}^{\frac{1}{j-1}} .
$$

Hence the proof is complete.

\section{References}

[1] A. Alb Lupaş, On special differential subordinations using Sălăgean and Ruscheweyh operators, Mathematical Inequalities and Applications, Volume 12, Issue 4, 2009, 781-790.

[2] A. Alb Lupaş, On a certain subclass of analytic functions defined by Salagean and Ruscheweyh operators, Journal of Mathematics and Applications, No. 31, 2009, 67-76.

[3] A. Alb Lupaş, A special comprehensive class of analytic functions defined by multiplier transformation, Journal of Computational Analysis and Applications, Vol. 12, No. 2, 2010, 387-395.

[4] A. Alb Lupaş, A new comprehensive class of analytic functions defined by multiplier transformation, Mathematical and Computer Modelling $\mathbf{5 4}$ (2011) 2355-2362.

[5] A. Alb Lupaş, On special differential subordinations using a generalized Sălăgean operator and Ruscheweyh derivative, Journal of Computational Analysis and Applications, Vol. 13, No.1, 2011, 98-107.

[6] A. Alb Lupaş, On a certain subclass of analytic functions defined by a generalized Sălăgean operator and Ruscheweyh derivative, Carpathian Journal of Mathematics, 28 (2012), No. 2, 183-190.

[7] A. Alb Lupaş, On special differential subordinations using multiplier transformation and Ruscheweyh derivative, Romai Journal, Vol. 6, Nr. 2, 2010, p. 1-14. 
PROPERTIES OA SUBCLASS OF UNIVALENT FUNCTIONS DEFINED BY USING A MULTIPLIER TRANSFORMATION AND RUSCHEWEYH DERIVATIVE

[8] A. Alb Lupaş, D. Breaz, On special differential superordinations using Sălăgean and Ruscheweyh operators, Geometric Function Theory and Applications' 2010 (Proc. of International Symposium, Sofia, 27-31 August 2010), 98-103.

[9] A. Alb Lupaş, Certain special differential superordinations using multiplier transformation, International Journal of Open Problems in Complex Analysis, Vol. 3, No. 2, July, 2011, 50-60.

[10] A. Alb Lupaş, On special differential superordinations using multiplier transformation, Journal of Computational Analysis and Applications, Vol. 13, No.1, 2011, 121-126.

[11] A. Alb Lupaş, On special differential superordinations using a generalized Sălăgean operator and Ruscheweyh derivative, Computers and Mathematics with Applications 61, 2011, 1048-1058, doi:10.1016/j.camwa.2010.12.055.

[12] A. Alb Lupaş, Certain special differential superordinations using a generalized Sălăgean operator and Ruscheweyh derivative, Analele Universitatii Oradea, Fasc. Matematica, Tom XVIII, 2011, 167-178.

[13] A. Alb Lupaş, On special differential superordinations using multiplier transformation and Ruscheweyh derivative, International Journal of Research and Reviews in Applied Sciences 9 (2), November 2011, 211-222.

[14] A. Alb Lupaş, Certain special differential superordinations using multiplier transformation and Ruscheweyh derivative, Journal of Computational Analysis and Applications, Vol. 13, No.1, 2011, 108-115.

[15] A. Alb Lupaş, Some differential subordinations using Ruscheweyh derivative and Sălăgean operator, Advances in Difference Equations.2013, 2013:150., DOI: 10.1186/1687-1847-2013-150.

[16] F.M. Al-Oboudi, On univalent functions defined by a generalized Sălăgean operator, Ind. J. Math. Math. Sci., 27 (2004), 1429-1436.

[17] L. Andrei, Differential subordinations using Ruscheweyh derivative and generalized Sălăgean operator, Advances in Difference Equation, 2013, 2013:252, DOI: 10.1186/1687-1847-2013-252.

[18] L. Andrei, V. Ionescu, Some differential superordinations using Ruscheweyh derivative and generalized Sălăgean operator, Journal of Computational Analysis and Applications, Vol. 17, No. 3, 2014, 437-444 
PROPERTIES OA SUBCLASS OF UNIVALENT FUNCTIONS DEFINED BY USING A MULTIPLIER TRANSFORMATION AND RUSCHEWEYH

[19] A.R. Juma, H. Zirar, Properties on a subclass of p-valent functions defined by new operator $V_{p}^{\lambda}$, Analele Univ. Oradea, Fasc. Math, Tom XXI (2014), Issue No. 1, 73-82.

[20] St. Ruscheweyh, New criteria for univalent functions, Proc. Amet. Math. Soc., 49(1975), 109-115.

[21] G. St. Sălăgean, Subclasses of univalent functions, Lecture Notes in Math., Springer Verlag, Berlin, 1013 (1983), 362-372.

Alina ALB LUPAŞ,

Department of Mathematics and Computer Science,

University of Oradea,

1 Universitatii Street, 410087, Oradea, Romania.

Email: dalb@uoradea.ro 